\title{
Mathematical incorrectnes of so called Higuchi's fractal dimension
}

\author{
Dalibor Martišek
}

Institute of Mathematics, Faculty of Mechanical Engineering, Brno University of Technology, 61669 Brno, Czech Republic

Correspondence: dmartisek@email.cz

\begin{abstract}
So called Higuchi's method of fractal dimension estimation is widely used and the term Higuchi's fractal dimension even occurs in many publications. This paper deals with this method from mathematical point of view. Terms distance and dimension and its basic properties are explained and Higuchi's dimension according the original source is defined. Definition of Higuchi's dimension was comparated with mathematical definition of the distance and dimension. It is showed, that the definition of the Higuchi's dimension does not satisfy axioms of distance and dimension. So called Higuchi's method and Higuchi's dimension are mathematically incorrect. Therefore, all results achieved by this method are scientifically unreliable.
\end{abstract}

Keywords: Higuchi's method, Higuchi's fractal dimension, distance, metric space

\section{Introduction}

Fractal dimension is any dimension that allows non-integer values. The oldest and the most general among them is the Hausdorff dimension (H-dimension). Nowadays, many other definitions are used as well.

Measurement of the fractal dimension plays an important role in many applications in engineering and science. The fractal dimension is measured either from time series or from digital images. The fractal dimension of various structures has been studied recently. [1] deals with the relation between the fractal dimension of surfaces of metal samples and their wear resistance coefficient as well as with the relation between the volume of pores in ceramics and the Hausdorff dimension of the pore boundary. [2] and [3] perform Hausdorff dimension analysis on fracture surfaces of porous materials such as hydrated cement pastes. The Hausdorff dimension of a fracture surface gives us information on the material and its properties. [4] studies the properties of different fracture surfaces (of metals, ceramics, rocks etc.) and their fractal properties. [5] studied the fracture surfaces of aluminium alloys subjected to four different heat treatments to find that their fractal dimensions were almost identical. [6] showed that the fractal dimension can be a measure of toughness in metals.

Many researchers have suggested using the fractal dimension to quantify rock joint roughness - see [7 -14] for example. There exist also many papers which deal with fractal dimension for other various purposes: natural phenomena [15], medicine [16], clinical neurophysiology especially [17], seismology [18], computer science [19] or communications technology [20].

Unfortunately, some published papers contain many inaccuracies and even mathematical nonsenses caused by insufficient mathematical foundations from which their authors deduce their conclusions - see [3, 20 - 28] for example. A special group of incorrect papers are texts which deal with so called Higuchi's method or Higuchi's fractal dimension - see [20, 29 - 35]. Research based on this incorrrect method was published even in physical [36], fractal [37] and chaos [38] journals

\section{Materials and Methods}

Each definition of a fractal dimension is based on the concept of distance or length of abscissa. In mathematics, they may be abstract terms and they can be defined in many ways. However, each distance or length must satisfy a few simple properties. Formally, metric space is defined in mathematics: A metric space is an ordered pair $(\mathcal{M} ; L)$ where 
$\mathcal{M}$ is a set and $L: \mathcal{M} \times \mathcal{M} \rightarrow \mathbb{R}$ is a mapping such that for any $x ; y ; z \in \mathcal{M}$, the following holds:

a) $L(x ; y) \geq 0$

b) $L(x ; y)=0$ if and only if $x=y$

c) $L(x ; y)=L(y ; x)$

d) $L(x ; z) \leq L(x ; y)+L(y ; z)$

Mapping $L$ is called distance function or metrics on set $\mathcal{M}$.

If we speak of any dimension, we obviously presume its integer value - objects are one-, two- or three dimensional. It is so called topological dimension. Fractal dimension is an arbitrary dimension which allows non-integer values. For its computing, we must measure a series of its approximations. For example, perimeter of a circle can be measured approximately with the series of hypothetical folding rules - polygons with shortenig segments. Each segment shortening in half means approximately twice as many segments. If we denote length of segments as $L_{k}$ and corresponding numer as $N_{k}$ then $N_{k} \cdot L_{k}^{1} \approx$ constant. Perimeter of a circle is one-dimensional. For measuring of its interior, square grid can be used by analogy. Each shortening of square side $L_{k}$ to one half means approximately quadruplicate number of squares $N_{k}$, i.e. $N_{k} \cdot L_{k}^{2} \approx$ constant. Interior of a circle is two-dimensional. For interior of a ball is $N_{k} \cdot L_{k}^{3} \approx$ constant by analogy. It is three-dimensional. Generally, this dimension is defined as the number $D$, for which is

$$
N_{k} \cdot L_{k}^{D} \approx \text { constant }
$$

However, this dimension is not topological because number $D$ can be non-integer for many geometric shapes. $D=1.2618 \ldots$ for so called Koch curve, $D=1.5849 \ldots$ for so called Sierpinski triangle e.t.c. It is possible to proof that each fractal dimension of each set is greater or equal to its topological dimension. Topological dimension of Koch curve and also Sierpinski triangle is equal to on efor example. See [39 - 41] for more information about these problems.

Higuchi's method [29] is a common method of the estimation of the fractal dimension of a curve The Higuchi's method is defined only for functions $X(t)$, whose values are known only in points $t=1 ; 2 ; \ldots ; M$. An approximation $X_{k}^{m}$ of the graph of function $X(t)$ are polygons going through points

$$
X_{k}^{m}: X(m) ; X(m+k) ; X(m+2 k) ; \ldots ; X\left(m+\left\lfloor\frac{N-m}{k}\right\rfloor \cdot k\right) ; m=1 ; 2 ; \ldots ; k
$$

and the "length" of the polygon is defined as

$$
L_{m}(k)=\left(\sum_{i=1}^{\left\lfloor\frac{M-m}{n}\right\rfloor}|X(m+i k)-X(m+(i-1) k)|\right) \cdot \frac{M-1}{\left\lfloor\frac{M-m}{n}\right\rfloor \cdot k^{2}}
$$

where [-] stands for the nearest lower integer [29].

Principle of this method is illustrated in Fig. 1 where

for simplicity.

$$
\Delta y_{i}=|X(m+i k)-X(m+(i-1) k)|
$$

The values of $L_{m}(k)$ are averaged over $m$ to obtain function $\langle L(k)\rangle$. Then Higuchi claims that if $\langle L(k)\rangle$ is proportional to $k^{-D}$, then the curve is a fractal with dimension $D$. However, each fractal dimension must work with concept of length or distance according to points a) -d) in previous text and must be equal or greater than topological dimension. We have verified whether the Higuchi's dimension has these properties or not. 


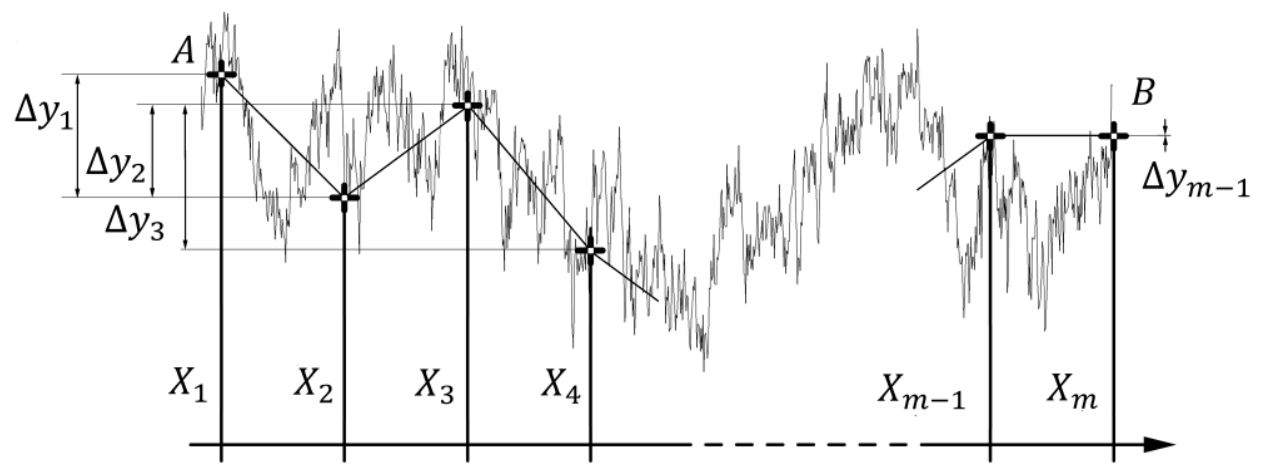

Figure 1. Principle of so called Higuchi's method.

\section{Results}

Higuchi tested his method on artificially generated noise (see Fig. 1) with good results. However, with the fractal dimension close to one (see Fig. 2), we get meaningless results.

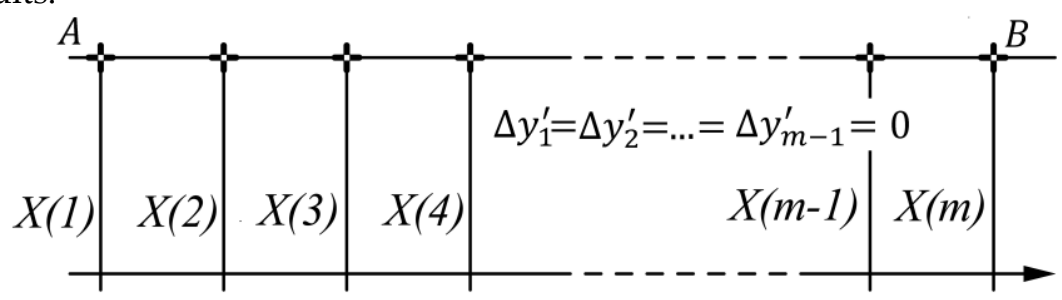

Figure. 2: Higuchi's method applied to a whole straight line gives incorrect result

If the Higuchi's method is applied to a constant time series

$X(1)=X(2)=\cdots=X(M)$,

we obtain $\Delta y_{i}=0$ for each $i$ in (3), therefore, sum of $\Delta y_{i}$ is equal to zero as well and $\quad L_{m}(k)=0$ for each $m, k$ in (2) and $L(A B)=0$ although points $A, B$ are not identical.

It is contrary to the condition b) in Sec. 2. Moreover, if $\langle L(k)\rangle$ is proportional to $k^{-D}$ (as Higuchi claims) then must be $D=0$ and fractal dimension would be smaller than topological dimension (it is equal to one). It means that so called Higuchi's dimension is contrary to known properties of a fractal dimension.

\section{Conclusion}

As was shown in previous section, so called Higuchi's method for fractal dimension measurement and also the term Higuchi's dimension is mathematically incorrect. Therefore, all results achieved by this method must be considered scientifically unreliable.

Funding: This research received no external funding

Acknowledgments: In this section, you can acknowledge any support given which is not covered by the author contribution or funding sections. This may include administrative and technical support, or donations in kind (e.g., materials used for experiments).

Conflicts of Interest: The authors declare no conflict of interest. 


\section{References}

1. Borodich, F. M.: Fractals and fractal scaling in fracture mechanics. International Journal of Fracture, 1999, Vol 95, pp. 239-259 DOI 10.1023/A:1018660604078

2. Ficker, T., Martišek, Jennings, M., H.: Roughness of Fracture Surfaces and Compressive Strength of Hydrated Cement Pastes, Cement and Concrete Research, 2010, Vol. 40, No. 6, pp. 947-955

3. Ficker, T. et al: Fracture Surfaces of Porous Materials, A Letters Journal Exploring the Frontiers of Physics, 2007, Vol. 80, No. 1, 16001604

4. Bouchaud, E., Lapasset, G., Planès, J.: Fractal Dimension of Fractured Surfaces: An Universal Value? Europhysics Letters (EPL), 1990, Vol. 13, pp. 73-79, DOI 10.1209/0295-5075/13/1/013

5. Cervantes-De la Torre, F. et al.: Fractal dimension algorithms and their application to time series associated with natural phenomena, J. Phys., 2013, Conf. Ser. 475012002

6. Mandelbrot, B. B., Passoja, D. E., Paullay, A. J.: Fractal character of fracture surfaces of metals. Nature, 1984, Vol. 308(5961), pp. 721-722, DOI 10.1038/308721a0

7. Brown, S. R. and Scholz, C. H.: Broad Band Width Study of the Topography of Natural Rock Surfaces, Journal of Geophysics Research, 1985, Vol. 90, pp. 12575-12582, https://doi.org/10.1029/JB090iB14p12575

8. Miller, S. M., McWilliams, P. C. and Kerkering, J. C.: Ambiguities in Estimating Fractal Dimensions of Rock Fracture Surfaces. In: Balkema, A.A., Ed., Proceedings of the 31st U.S. Symposium, Colorado School of Mines, Rotterdam, June 18-20, 1990, pp. 471-478.

9. Power, W. L. and Tullis, T. E.: Euclidean and Fractal Models for the Description of Rock Surface Roughness. Journal of Geophysical Research, 1991, Vol 96, pp. 415-424, https://doi.org/10.1029/90JB02107

10. Huang, S.L., Oelfke, S.M. and Speck, R.C.: Applicability of Fractal Characterization and Modeling to Rock Joint Profiles. International Journal of Rock Mechanics and Mining Science, 1992, Vol. 29, pp. 89-98. https://doi.org/10.1016/0148-9062(92)92120-2

11. Poon, C.Y., Sayles, R.S. and Jones, T.A.: Surface Measurement and Fractal, Characterization of Naturally Fractured Rocks. Journal of Physics D: Applied Physics, 1992, Vol. 25, pp. 1269-1275. https://doi.org/10.1088/0022-3727/25/8/019

12. Odling, N.E.: Natural Fracture Profiles, Fractal Dimension and Joint Roughness Coefficients. Rock Mechanics, 1994, Vol. 27, pp. 135-153, https://doi.org/10.1007/BF01020307

13. Den Outer, A., Kaashoek, J. F., Hack, H. R. G. K.: Difficulties with Using Continuous Fractal Theory for Discontinuity Surfaces, International Journal of Rock Mechanics and Mining Science, 1995, Vol. 32, pp. 3-10. https://doi.org/10.1016/0148-9062(94)00025-

14. Ficker, T.: Fractal Properties of Rock Joint Coefficients, International Journal of Rock Mechanics E Mining Science, 2016, Vol. 94, pp. 27-31, https://doi.org/10.1016/j.ijrmms.2017.02.014

15. Mandelbrot, B. B.: The Fractal Geometry of Nature, W. H. Freeman and Company, New York, 1983

16. Fuss, F, K.: A Robust Algorithm for Optimisation and Customisation of Fractal Dimensions of Time Series Modified by Nonlinearly Scaling Their Time Derivatives: Mathematical Theory and Practical Applications, Computational and Mathematical Methods in Medicine, 2013, Volume, 19 pages, Article ID 178476

17. Kesić, S., Spasić, S. Z.: Application of Higuchi's fractal dimension from basic to clinical neurophysiology: A review. Comput Methods Programs Biomed. 2016 Sep. 133:55-70. doi: 10.1016/j.cmpb.2016.05.014. Epub 2016 May 30.

18. Galvez, G., et al. Parameters of Higuchi's method to characterize primary waves in some seismograms from the Mexican subduction zone, June 2012 Acta Geophysica 60(3) DOI10.2478/s11600-012-0033-9

19. Güçlü, U., Güçlütürk, Y., Chu Kiong Loo: Evaluation of fractal dimension estimation methods for feature extraction in motor imagery based brain computer interface Procedia Computer Science, 2011,Volume 3, Pages 589-594

20. Phothisonothai, M., Yasunori A., Watanabe, K.: Effects of time windowing for extraction of expression from Japanese speech: Higuchi's fractal dimension Communications and Information Technologies (ISCIT), 2013 13th International Symposium on Communications and Information Technologies (ISCIT), Page(s): 665-668

21. Brown, S. R., Scholz, C. H.: Broad Band Width Study of the Topography of Natural Rock Surfaces. Journal of Geophysics Research, 1985, Vol. 90, pp.12575-12582, https://doi.org/10.1029/JB090iB14p12575

22. Miller, S. M., McWilliams, P. C., Kerkering, J. C.: Ambiguities in Estimating Fractal Dimensions of Rock Fracture Surfaces. In: Balkema, A.A., Ed., Proceedings of the 31st U. S. Symposium, Colorado School of Mines, Rotterdam, June 18-20 1990, 471-478.

23. Power, W. L., Tullis, T. E.: Euclidean and Fractal Models for the Description of Rock Surface Roughness. Journal of Geophysical Research, 1991, Vol. 96, pp. 415-424. https://doi.org/10.1029/90JB02107

24. Huang, S. L., Oelfke, S. M., Speck, R. C.: Applicability of Fractal Characterization and Modeling to Rock Joint Profiles. International Journal of Rock Mechanics and Mining Science, 1992, Vol. 29, pp. 89-98. https://doi.org/10.1016/0148-9062(92)92120-2

25. Poon, C.Y., Sayles, R.S. and Jones, T. A.: Surface Measurement and Fractal Characterization of Naturally Fractured Rocks. Journal of Physics D: Applied Physics, 1992, Vol. 25, pp. 1269-1275. https://doi.org/10.1088/0022-3727/25/8/019

26. Odling, N. E.: Natural Fracture Profiles, Fractal Dimension and Joint Roughness Coefficients. Rock Mechanics, 1994, Vol. 27. pp. 135-153. https://doi.org/10.1007/BF01020307

27. Den Outer, A., Kaashoek, J. F., Hack, H. R. G. K. (1995). Difficulties with Using Continuous Fractal Theory for Discontinuity Surfaces. International Journal of Rock Mechanics and Mining Science, 1995, Vol. 32, pp. 3-10. https://doi.org/10.1016/01489062(94)00025-X

28. Ficker, T.: Fractal Properties of Rock Joint Coefficients. International Journal of Rock Mechanics E Mining Science, 2016, Vol. 94, pp. 27-31.

29. Higuchi, T.: Approach to an Irregular Time Series on the Basis of the Fractal Theory. Physica D, 1988, Vol. 31, pp. $277-283$. 
30. Gomolka, R. et al: Higuchi Fractal Dimension of Heart Rate Variability During Percutaneous Auricular Vagus Nerve Stimulation in Healthy and Diabetic Subjects FRONTIERS IN PHYSIOLOGY, 2018, Vol. 9, Article Number: 1162

31. Kesic, S., Spasic, S: Application of Higuchi's fractal dimension from basic to clinical neurophysiology: A review, Computer methods and programs in biomedicine, 2016, Volume: 133 Pages: 55-70

32. Kesic, S., et al: Ouabain modulation of snail Br neuron bursting activity after the exposure to $10 \mathrm{mT}$ static magnetic field revealed by Higuchi fractal dimension General physiology and biophysics, 2014, Volume: 33, Issue: 3, Pages: 335-344

33. Topcu, C. et al: Higuchi Fractal Dimension Analysis of Surface EMG Signals and Determination of Active Electrode Positions, 18th National Biomedical Engineering Meeting (BIYOMUT) Location: Istanbul, TURKEY Date: OCT 16-17, 2014

34. Bachmann, M. et al: Spectral Asymmetry and Higuchi's Fractal Dimension Measures of Depression Electroencephalogram, Computational and mathematical methods in medicine, 2013, article Number: UNSP 251638

35. Kalauzi, A. et al: Modeling the relationship between Higuchi's fractal dimension and Fourier spectra of physiological signals, Medical \& biological engineering $\mathcal{E}$ computing, 2012 Volume: 50, Issue: 7, Pages: 689-699

36. Bordin, L. et al: Tensor squeezed limits and the Higuchi bound, Journal of cosmology and astroparticle physics, Vol 2016, Issue: 9 , Article Number: 041

37. Rani, T. G. et al: Complex patterns in financial time series through Higuchi's fractal dimension, Fractal-Complex geometry patterns and scaling in nature and society, 2016, Vol. 24, Issue: 4, Article Number: 1650048, Published: DEC 2016

38. Ahammer, $\mathrm{H}$, et al: Is a two-dimensional generalization of the Higuchi algorithm really necessary? CHAOS, 2015, Volume: 25 Issue: 7, Article Number: 073104

39. Edgar, G.: Measure, topology and fractal geometry, Springer, pp. 272, 2008

40. Falconer, K. J.: Fractal geometry, Mathematical Foundation and Application, Second Edition, University of St. Andrews, 2003

41. Falconer, K. J.: The Geometry of Fractal Sets, Cambridge University Press, Cambridge, United Kingdom 1985. 